\title{
Visual Guides for Comprehending Digital Ink in Distortion Lenses
}

\author{
Paul Schmieder, Andrew Luxton-Reilly, Beryl Plimmer \\ Department of Computer Science \\ University of Auckland \\ Private Bag 92019 \\ Auckland, New Zealand \\ John Hosking \\ ANU College of Engineering and Computer \\ Science \\ Australian National University \\ Canberra, ACT 0200 \\ Australia \\ psch068@aucklanduni.ac.nz, [beryl, andrew]@cs.auckland.ac.nz, john.hosking@anu.edu.au
}

\begin{abstract}
We devised and tested two new visual guides to help users comprehend distorted sketched information in magnification lenses. Distortion techniques, such as fisheye lenses, have the advantage of magnifying information without occluding the surrounding content. However distorted information in the transition region requires extra mental workload to understand: this can lead to frustration and rejection of magnification lenses. Our evaluation shows any visual guide is better than none and identifies strengths and weaknesses of the new guides. We tested for the four visual properties important for understanding distorted information: scale, alignment, distance and direction. Surprisingly grids are not as effective in many contexts as our new lenses.
\end{abstract}

Focus + context strategies, comprehension of visual distortions, visual guides

\section{INTRODUCTION}

We investigate how visual guides can be used to support comprehension of visual distortions caused by, for example, a fisheye lens (Fig. 1). We use sketching as a context as it allows for rapid and unconstraint content creation and annotation (Agrawala and Shilman 2005) and is increasingly available on small displays. Distortion based lenses allow people to see parts of the content in greater detail without occluding other parts, but understanding the distorted content increases mental workload. Consider Fig. 1b and Fig. 1c, the area that is most difficult to comprehend is the transition region (shaded grey): yet these visual transformations are the essence of distortion techniques. We hypothesized that additional information such as displayed in the background in Fig. 1b\&c provide visual guidance that make distortions easier to comprehend. We describe two novel visual guides, foreground and background. The evaluation of these guides against a control condition and a traditional grid measures how each condition effects users' judgments of scale, alignment, distance and direction of distorted objects in the transition region.

It is essential to be able to magnify information on small displays. Different strategies, such as zooming interfaces, allow viewing of parts of the information in greater detail. The space required to display parts of the content enlarged means other parts are occluded. In a zooming interface, such as Google Maps, extra navigation (e.g. panning) is

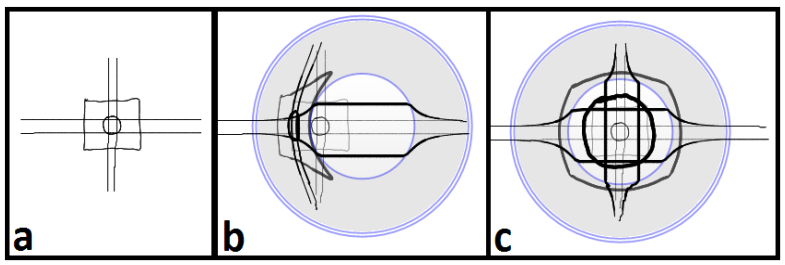

Figure 1: A rectangle surrounding a circle and 4 lines (a) covered by a fisheye lens and background guide $(b+c)$. The lens borders are coloured blue and the transition region grey.

required to view the excluded parts. People must form a mental image of all the information in their working memory which increases the mental workload (Wickens and Hollands 2000). These additional steps can also be frustrating (Grudin 2001). Optimal comprehension requires all pertinent information to be visible all the time.

Distortion techniques, also called focus + context strategies, do not occlude content. The advantages of the non-occluding strategies is demonstrated in (Lank and Phan 2004) where zooming interfaces are compared to fisheye lenses and in (Schmieder, Plimmer et al. 2012) where distortion lenses are used for content creation on small displays.

Distortion techniques offer promise for small displays, however understanding visually transformed information is hard (Grudin 2001). To recognize objects, distorted or not, their basic visual properties such as length, direction, angle and scale have to be correctly assessed (Cleveland and McGill 1984). We refer to these properties as orientation properties. As distortions change the 
visual appearance of objects, the assessment of orientation properties becomes more difficult. Prior work on user comprehension of distortions includes: use of a grid and/or shading (Zanella, Carpendale et al. 2002) and allowing users to define distorted regions to be displayed undistorted on an inset (Brosz, Carpendale et al. 2011). While these approaches help to better understand distortions overall, it is unclear how well they support the different aspects of orientation and if other approaches may be more suitable. As we will show, the efficacy of the visual guides (and grids), varies widely when judging orientation properties.

To address the difficulties involved in understanding visual distortions, we answer the following research questions: (a) To what extent do distortion based visualizations make orientation properties incomprehensible? (b) To what extent can visual guides help alleviate the distortion effects? By answering these questions, this paper makes three contributions to distortion techniques. First, we present new visual guides that afford better comprehension of distorted information. Second, by comparing our new guides, a grid and a no-help condition, the effects of different guiding strategies on comprehending visual distortions is gauged and their benefits are demonstrated. Third, we recommend to designers the visual guide options suitable for particular contexts.

\section{RELATED WORK}

Magnification interfaces are commonly grouped into three categories: overview+detail; zooming; and distortion based interfaces (Cockburn, Karlson et al. 2009). One main difference between them is whether they occlude content during magnification. Overview+detail and zooming interfaces spatially or temporally occlude content: additional space to display the magnified content replaces some of the unmagnified content. Distortion techniques visually transform content to generate space required for magnification, thus they don't occlude content but change its representation.

With non-occluding techniques the representation of the transformed content may be skewed (e.g. fisheye lens (Furnas 1986)), blurred (Kosara, Miksch et al. 2001) or made transparent (Pietriga and Appert 2008). Fisheye lenses (Furnas 1986) are the most common distortion technique. A fisheye lens consists of a central magnified region in which the content is equally magnified. This is surrounded by a distorted region that transitions between the magnified and non-magnified regions.

Distortion lenses have been applied to a variety of domains and tasks with varying success. Domains include maps (Pattath, Ebert et al. 2009), document navigation (Cockburn, Savage et al. 2005) and reading comprehension (Hornbaek and Frokjaer 2003). Target acquisition (Ramos, Cockburn et al.
2007) and large steering tasks (Gutwin and Skopik 2003) benefit from distortion lenses while performance on menu selection tasks decreases (Bederson 2000). Distortion based magnification lenses have been successfully used to create and edit content (Schmieder, Plimmer et al. 2012) but results indicate more user support may be useful.

Several problems have been identified with distortion lenses. Target acquisition is one of the problems caused by scale differences between motor and visual space in the magnified region (Gutwin 2002). Strategies to overcome this problem include pinning the lens (Schmieder, Plimmer et al. 2012) and lens flattening (Gutwin 2002). Another problem, and the focus of this work, is the additional mental workload required to comprehend and interact with the distorted information in the transition region. The distorted objects and their relationships to other objects distorted, magnified or non-magnified, can be difficult to judge (Carpendale, Cowperthwaite et al. 1997).

The general perception of visual objects' properties in relation to their actual properties is the discipline of psychophysics (Wagner 2006). Bertin (1977) and Cleveland and McGill (1984) believe that visual variables (Bertin) or elementary perceptual tasks (Cleveland and McGill) are used to extract information from visual data. Cleveland and McGill extended Bertin's original seven visual variables (position, size, shape, value, colour, orientation and texture) into ten elementary perceptual tasks (position common scale, position non-aligned scale, length, direction, angle, area, volume, curvature, shading, and colour saturation) as they believe that some of Bertin's categories were too broad and they also split Bertin's variables along perceptual lines. Wigdor, Shen et al. (2007) and Bezerianos and Isenberg (2012) use a subsets of Cleveland and McGill's elementary perceptual tasks to inform their work on visual distortions and to provide guidelines helping to overcome such distortions in the context of varying distances and viewing angles in tabletop environments.

Visual guides can help navigation and understanding of distorted and non-distorted content. Jul and Furnas (1998) propose view-based guides, such as landmarks, to navigate multiscale worlds. Zanella et al. (2002) investigated the use of a cartographic grid and shading individually and combined on maps and found the grid to be the most useful visual cue. Zanella et al.'s goal was to help people to identify distorted regions and make basic judgments but not to help them to understand the distorted content.

The Undistort Lens (Brosz, Carpendale et al. 2011) supports distortion comprehension by reversing the distortions. It allows users to specify an area in the distorted region to be displayed undistorted in an inset. Their study demonstrated various scenarios 


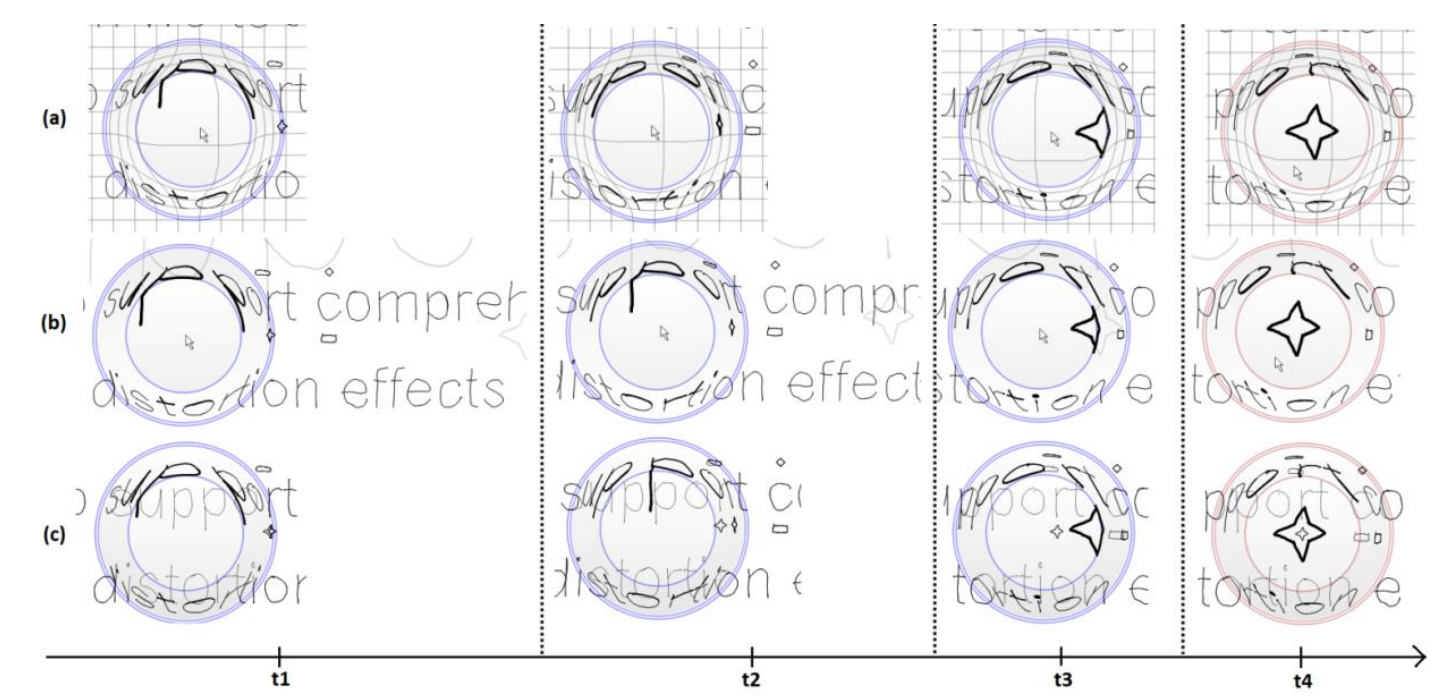

Figure 2: The distortion guides' behaviours when freely moving (t1-t3) and pinned down (t4). (a) The grid lines and content covered by the lens. (b) The foreground guide displays the star magnified and undistorted on the right side as the star enters the transition region until the distorted and magnified views merge as the star enters the magnified region ( $t 3$ \& t4).

(c) When the star enters the transition region with the background guide (t1) the undistorted, unmagnified view is shown under the lens area with the distorted view overlaid. The template used for these scenarios is shown in Fig. 3 (a).

in which this lens helps to better understand the distorted content. While the Undistort Lens is a valuable addition to complement distortion based techniques, it is not a visual guide, as it requires user interaction to define its region of effect.

In sum, distortion-based techniques are a valuable magnification strategy to avoid content occlusion. Yet, they make comprehending the distorted content more difficult. In particular, judgments regarding elementary perceptual tasks (Cleveland and McGill 1984) are impeded. Research has shown that using visual guides helps people to locate distortions and make basic judgments in terms of the distortion levels. However, to our knowledge there is no work investigating how people can be supported to better comprehend visually distorted information; i.e. to be able to identify a transformed object based on its distorted representation. Nor are there studies specifically investigating the impact of distortions on each of the orientation properties individually.

\section{VISUAL GUIDES FOR DISTORTION COMPREHENSION}

We developed two novel visual guides that support comprehension of distorted objects and implemented a grid. Fig. 2 shows a time sequence of each guide in a fisheye lens, a small star appears at the outer border of the lens in $\mathrm{t} 1$ (below the ' $r$ ') and moves through the transition region to the center of the lens in $t 4$. The undistorted text is in Fig. 3a. The three visual guides are explained in the following sections.

\subsection{Grid}

The idea of a grid is to provide the participants with a frame of reference. By analysing how the gridlines behave a person may be able to deduce a distorted shape's original representation. The grid, a grating of equally spaced crossed bars in the background (Fig. 2a), is semi opaque to differentiate it from the canvas content. The same distortion is applied to the grid lines and content. We tested different grid sizes; the difficulty was to provide a sufficiently fine-grained grid while minimizing distraction. We determined that having one grid cell fitting well inside the focus region gave the best trade-off between detail and distraction.

\subsection{Foreground}

The motivation for the foreground technique is to show an additional view of distorted objects. The lens shows magnified content in the focus region and the distorted content in the transition region. Additionally, a magnified version of the transition region content is show in the foreground. A shape in the transition region is shown distorted and semi opaque in the transition region and its magnified version shown fully opaque in the foreground (Fig. 2 (b): t3-4). The content in the foreground guide spreads beyond the borders of the lens.

\subsection{Background}

The motivation for the background lens is to show distorted content undistorted under the lens. Thus users have both views in the same location so that they do not have to change perspective as with the foreground lens. The background guide shows the distorted view plus the shapes in their original position and size in the background of both the magnified and transition regions of the lens (Fig. 
2c). When a shape is in the lens's midpoint (Fig. 2c - t4) both its views are on top of each other. If a user adds an object to the magnification region it is instantly duplicated in the background.

\section{EVALUATION OF THE VISUAL GUIDES}

We ran an experiment with the three visual guides and a control condition. The aim was to gauge the support the guides provide and to compare the techniques against each other and no guidance. We were interested in the general performance of the guides and also their effect on comprehension of scale, alignment, direction and distance. These orientation properties are based on the four elementary perceptual tasks of Cleveland and McGill's (1984) work namely; area (scale), angle (alignment), direction and length (distance). We chose those four for two reasons: first, Cleveland and McGill's ranking (1984) lists them among the best elementary perceptual tasks for accurate judgments and, second, because we hypothesize them to be influenced by the visual distortions. We did not test position as the head can be moved freely over the display so that it is always centred above the lens. Slope was not included as previous work suggests it is closely related to angle (Cleveland and McGill 1985). Colour is ignored as all our drawings are in grayscale.

We configured a distortion lens based on the fisheye technique (Furnas 1986; Sarkar and Brown 1992) as it is commonly used and hence the knowledge gained will be readily applicable to existing work in distortion-based interfaces. The lens regions were shaded light grey with blue borders (Fig. 2t1-t3) to outline the focus region as well as the complete lens. When pinned down, the blue lens borders were replaced by red ones (Fig. 2: t4). The magnification factor was set to four.

\subsection{Method}

Our study had a within-subject design, which tested one factor: visual guide (Control, Grid, Foreground and Background). Participants completed five tasks using all three guides for each task. They completed one task with all three guides and without any visual help (control condition) before commencing the next task. The task order was identical: every participant started with task one and finished with task five but to reduce the learning effect the order of guides within tasks was counter balanced. Furthermore there were four versions of each task; e.g. the four clocks in task two showed different times in each task version. The task order was kept identical because, first, the first three tasks were completed with a static lens accustoming the participants to visual distortion and, second, we hypothesized that the order of guides has a bigger impact on learning effect. Depending on the orientation property of interest a task may be completed on a static image or in the interactive test environment. To test all four orientation properties together (task 1), alignment (task 2) and scale (task 3) the lens' position was required to be identical to ensure the same distortion effects for the same objects across all participants: working with static images guaranteed these positioning requirements. As the primary goal of this evaluation is to gauge the effects of visual guides on distortions and not the guides' usability, static images were favoured over using the test environment for tasks 1-3. The interactive environment was used for tasks 4-5.

\subsection{Participants}

There were 24 participants, 10 females and 14 males, ranging in age from 19 to 32 years. Four participants were left-handed. 20 participants were students from different majors and the remaining 4 worked full time. Most had no experience of distortion effects, but 3 had used them in other contexts such as photography.

\subsection{Apparatus}

A $6 \mathrm{~cm}$ lens covered the area of interest on the template image shown in Fig. 3a. For all tasks participants used a HP EliteBook 2740p tablet PC with a 13" display of resolution $1280 \times 800$. The tablet has an Intel ${ }^{\circledR}$ core ${ }^{\text {TM }}$ i7 $\mathrm{CPU}$ with $2.67 \mathrm{GHz}$ and 4GB RAM. The HP digital stylus that came with the tablet was used for interaction. For tasks 1-3 the participants were presented with a static image and the stylus used for recording answers. For tasks $4 \& 5$ participants used the test environment and stylus. Sessions were recorded using screen capture and sound.

The fisheye lens and visual guides were implemented in our test environment. The test environment is written in C\# and uses the Microsoft Windows Real-Time Stylus interface. Following common practice (Jakobsen and Hornbaek 2011), the lens's flat focus region was set to two-thirds of the lens diameter and the transition region took the balance of the space. The implementation is identical to Schmieder et al's (2012).

The stylus is used to capture input on the tablet. The fisheye lens if not stated otherwise follows the stylus. The stylus can either hover over the tablet surface to navigate the canvas or touch the surface to draw. The stylus button is used to pin and unpin the lens thus decoupling and coupling stylus and lens movement. If decoupled the participants can move the stylus freely without the lens following but they can only draw in the focus region. The zoom level is set to four.

\subsection{Tasks}

Participants completed each of the five tasks using the three guides: (grid, foreground and 


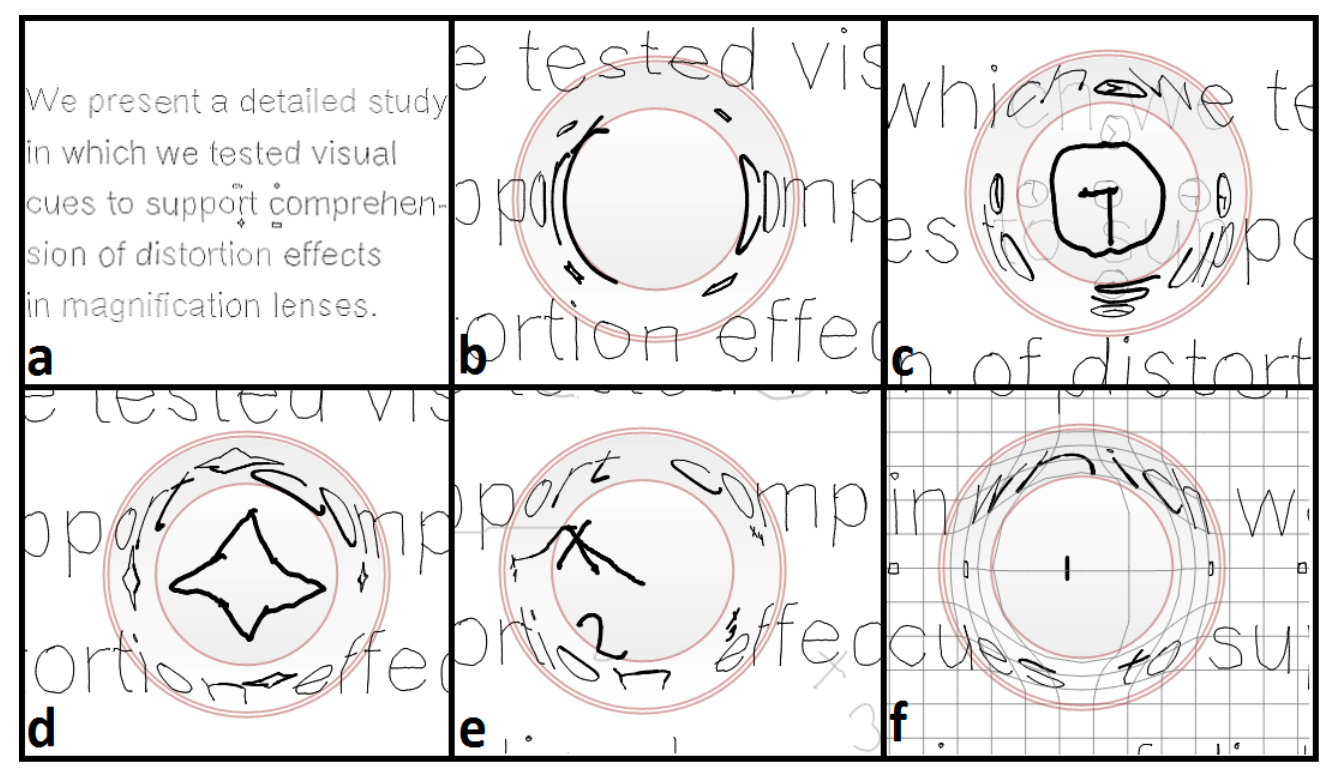

Figure 3: (a) The background used for all the tasks with the template used for task 1 (see (b)). (b) The first task testing alignment, scale, direction and distance with the control condition. (c) Task 2 testing alignment with the background guide. (d) Task 3 testing scale with the control condition. (e) Task 4 testing direction with the foreground guide. (f) Task 5 testing distance with the grid.

background) and the control condition. Each task used a different template but all had text in the background to simulate a busy canvas. The single spacing text and the templates for each task were hand drawn by the researcher. For the first three tasks involving static images showing a scenario, participants wrote the solution on the image. For the remaining two tasks, a template was provided which had to be completed and participants could only draw in the focus region. The lens was pinned while drawing so the situation was the same as when using the static images.

The tasks were designed with the aim of specifically testing the impact of distortions on scale, alignment, direction and distance (Table 1). While it is hard if not impossible to test any of the four elements in isolation, tasks two to five each focus on one element. Task 1 targets all four areas at once.

Task 1, the general task, asked participants to identify objects in the lens's transition region (for example clockwise from 12 o'clock Fig. 3 (a \& b) has a diamond, rectangle, star, and ellipse). All four task versions included four out of twelve basic shapes or words that had to be identified. Participants were advised that there were no identical shapes; e.g. only one of the four could be a circle. This task tests all four orientation properties. For example, when identifying a distorted square (Fig. 1) it is important to recognize that the opposite edges are equally long (scale), all edges form right angles (alignment) and opposite edges are parallel (direction). Finally to determine it is a square as opposed to a rectangle each side has equal length (distance).
Task 2 tested alignment (Fig. 3 (c)). Participants had to read the time on the four analogue clocks in the transition region. While this is an unlikely scenario, reading distorted clocks allowed us to specifically measure the distortions' impact on alignment. Participants were advised that the times would be in multiples of five; e.g. the clock may show $4: 15$ or $4: 20$ but not $4: 18$.

Task 3 tested relative and absolute scale judgments (Fig. 3 (d)). The participants were asked to judge shape sizes. The image had five identical shapes (e.g. diamonds): four in the transition region and one in the focus region. The task was to order the shapes in the transition region according to size starting with the smallest. Participants also had to identify the shape in the transition region that was of the same size as the shape shown in the lens's focus region.

Task 4 is designed to test the impact of distortion on direction (Fig. 3 (e)). Participants had to connect four crosses with straight lines. The template showed four numbered crosses which had to be connected in ascending order. To connect crosses, the lens had to be pinned over the starting cross. The distances between the lines required participants to reposition their lens as the pairs were too far away to be in the focus region at the same time. We judged a line to be sufficiently straight if the direction from the start cross pointed at the target cross.

Task 5 primarily required judgment of distance (Fig. 3 (f)). Participants had to add two shapes to a line of existing shapes. The goal was to add shapes of the same size equidistant to two existing shapes. Participants had to position the lens between existing shapes and pin it before starting to draw. If 
the center point of the participants shapes was within $5 \%$ of the exact midpoint between the two surrounding shapes and within $5 \%$ of their size the task was regarded as correct.

\subsection{Procedure}

Participants were seated at a table with the tablet immediately in front of them. A tutorial was given to guide them through the experiment. After reading the general instructions the participants completed the first section of the questionnaire. Then the study facilitator explained the fisheye lens as well as all three visual guides.

Before each task the participants read a task description in the tutorial advising them what to do. Each task consisted of four subtasks each with a different visual guide. Once a task was completed, the participant completed the corresponding part of the questionnaire. These steps were repeated for each of the five tasks.

After all tasks a final section of the questionnaire was completed. The experiment concluded with a (recorded) discussion between participant and facilitator about each guide's pros and cons. It took the participants on average 36 minutes to complete the study.

\subsection{Data Captured}

Completion time and error rate were measured for each task. Completion time is defined as the time span between a participant being shown a screenshot and completing the task (tasks 1-3) and between a participant starting to draw till finishing (tasks 4-5).

A questionnaire comprising three parts was used to capture different aspects of the participants' interaction. The first part gauged participants' familiarity with digital sketching and distortion techniques. The second part asked participants to rate the visual guides according to their helpfulness and distraction on a 5 point Likert scale. It also asked the participants to rank the lenses according to preference. This last part asked the participant to, first, provide an aggregate ranking and, second, to rate the guides on a 10-point scale.

\section{RESULTS}

Here we report mean scores for each specific task followed by a summary of task results, participant's rankings, and ratings of the guides. We also report participants' comments and the study facilitator's observations.

Completion time is normally distributed while error rate and questionnaire data are not. To analyse the completion time we use a one-way repeatedmeasures ANOVA. To further examine significant differences between the variables, Bonferroni-
Table 1: The tasks, the distortion property they are designed to test:

\begin{tabular}{|l|l|}
\hline Task & Property \\
\hline 1 - Identify the Shape & Alignment, Scale, Direction, Distance \\
\hline 2 - What's the time & Alignment \\
\hline 3 - What's the size & Scale \\
\hline 4 - Draw straight lines & Direction \\
\hline 5 - Fill the gaps & Distance \\
\hline
\end{tabular}

corrected pairwise comparisons are used. Greenhouse-Geisser estimates are used for correction if sphericity is violated. To analyse the error rate and questionnaire data, Friedman's ANOVA (nonparametric) tests are used. For further analysis investigating the differences between variables, Bonferroni-corrected Wilcoxon signedrank tests are used and the results corrected for the number of tests. All effects are reported with adjusted $p$-values $(p=0.05$ divided by the number of tests). The number of tests is six for the error rate, ranking and rating and three for helpfulness and distraction. No learning effects in the form of statistically significant differences were found between the tasks and error rate and/or completion time.

\subsection{Scale, Alignment, Direction and Distance}

The tasks are designed to address four shape properties which distortions disrupt: scale, alignment, direction and distance The following statistical analysis looks at each of them in turn in terms of time, error rate (Fig. 4), preference (Fig. 5), distraction and helpfulness.

The first task testing all four orientation properties shows a significant difference for completion time $(F(3,69)=7.622 ; p=0.010)$. Pairwise comparisons show the mean grid time $(27.08 \mathrm{~s} \pm 10.9)$ was significantly slower than the foreground (18.29s $\pm 4.9 ; \quad p=0.013$ ) and background guides (18.29s $\pm 5.8 ; p=0.014)$.

The error rate shows a significant difference $\left(x^{2}(3)=39.431 ; p<0.001\right)$ where the control condition $(1.54 \pm 1.1)$ results in significantly more errors than any guide; grid $(0.79 \pm 0.78 ; p=0.013)$, foreground guide $(0.08 \pm 0.28 ; p<0.001)$, background guide $(0.21 \pm 0.41 ; p<0.001)$. Additionally, using the grid produces significantly more errors than foreground $(p=0.001)$ and background guides $(p=0.002)$.

The analysis of the questionnaires shows a significant difference for helpfulness $\left(x^{2}(2)=27.31\right.$; $p<0.001)$ and distraction $\left(x^{2}(2)=11.914 ; p=0.003\right)$. Compared to the grid $(2.21 \pm 1.48)$ the foreground (4.71 $\pm 0.46 ; p<0.001)$ and the background guides $(4.38 \pm 0.71, p<0.001)$ are on average significantly more helpful. However, the comparisons also shows that the grid $(1.63 \pm 0.82)$ is significantly less distracting than the foreground guide (2.67 \pm 1.34 ; $\mathrm{p}=0.004$ ) but is not significantly different to the 
background guide $(2.29 \pm 1.12)$. In summary, the background and foreground guides resulted in significantly less errors and were rated as more helpful than grid and control condition. However the grid was less distracting than the foreground guide.

\subsubsection{Alignment}

Task 2 tested alignment. The results show significant differences for time $(F(3,69)=4.651$; $p=0.005)$ and error rate $\left(x^{2}(3)=33.721 ; p<0.001\right)$. Pairwise comparisons show the grid $(34.26 s \pm 11.4)$ is significantly slower than the foreground guide (27.92s $\pm 7 ; p=0.015)$. The comparisons also shows that, first, having no visual guide $(1.46 \pm 0.88)$ resulted in significantly more errors than foreground $(0.38 \pm 0.58 ; \quad p<0.001)$ and background guides $(0.33 \pm 0.48 ; \quad p<0.001)$ and, second, the grid $(1.33 \pm 0.87)$ also results in significantly more errors than foreground $(p<0.001)$ and background guides $(p=0.001)$.

There is a significant difference for helpfulness $\left(x^{2}(2)=39.747 ; p<0.001\right)$ and none for distraction. Pairwise comparisons show that the grid $(2.04 \pm 1.16)$ is significantly less helpful than foreground $(4.88 \pm 0.34 ; p<0.001)$ and background guides $(4.17 \pm 0.76 ; \quad p<0.001)$. The background guide is also significantly less helpful than foreground guide $(p=0.001)$. In summary the grid and control condition resulted in more errors while background and foreground performed equally but the foreground was perceived as more helpful.

\subsubsection{Scale}

Task 3 tested scale. The results show no significant differences for time but one for error rate $\left(x^{2}(3)=27.39 ; \quad p<0.001\right)$. Pairwise comparisons show that the control condition $(0.79 \pm 0.59)$ results in significantly more errors than foreground $(0.13 \pm 0.34 ; \quad p<0.001)$ and background guides $(0.21 \pm 0.41 ; p=0.001)$. The grid $(0.67 \pm 0.48)$ also results in significantly more errors than foreground $(\mathrm{p}=0.002)$ and background guides $(\mathrm{p}=0.001)$.

Looking only at the errors made during sorting the shapes according to size no significant difference can be found. However, when matching the shape in the magnified region to its equal in the transition region a significant difference can be found $\left(x^{2}(3)=26.753 ; \quad p<0.001\right)$. Pairwise comparisons show the same pattern as with the total errors made for task 3: first, the control condition $(0.71 \pm 0.46)$ results in significantly more errors than foreground $(0.08 \pm 0.28 ; p<0.001)$ and background guides $(0.21 \pm 0.41 ; p=0.001)$ and, second, grid $(0.63 \pm 0.49)$ results in significantly more errors than foreground $(p=0.002)$ and background guides $(p=0.002)$.

There is a significant difference for helpfulness $\left(x^{2}(2)=23.4 ; \quad p<0.001\right)$ and none for distraction. Pairwise comparisons show that the grid

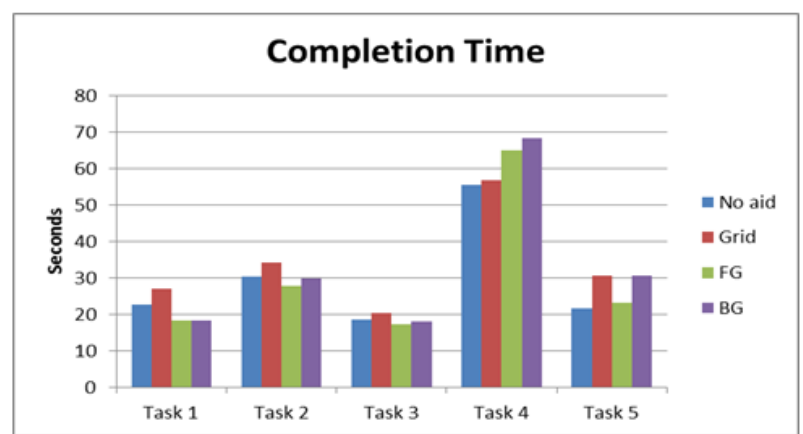

Figure 4: The completion time means for the 5 tasks and the visual support: none, grid, foreground (FG) and background (BG)

$(3.21 \pm 1.14)$ is significantly less helpful than foreground $(4.58 \pm 0.5 ; p<0.001)$ and background guides $(4.58 \pm 0.5 ; \quad \mathrm{p}=0.001)$. In summary, foreground and background guides produce the best results for scale as they result in significantly fewer errors and are the most helpful.

\subsubsection{Direction}

Task 4 tested direction. The results show significant differences for time $(F(3,69)=11.763$; $\mathrm{p}<0.001)$ and error rate $\left(x^{2}(3)=22.66, p<0.001\right)$. Pairwise comparisons show the background guide $(68.38 \mathrm{~s} \pm 22.3)$ is significantly slower than the control condition $(55.5 \mathrm{~s} \pm 15.31 ; \mathrm{p}=0.001)$ and grid (56.79s $\pm 16.83 ; \mathrm{p}=0.004)$. The control condition $(2.04 \pm 0.75)$ results in significantly more errors than using the grid $(1.04 \pm 1.04 ; p<0.001)$, foreground $(1.13 \pm 0.74, \quad p=0.001)$ and background guides $(1.46 \pm 0.78 ; p=0.006)$.

While no significant effect for helpfulness could be found one for distraction was found $\left(X^{2}(2)=16.029\right.$; $p<0.001)$. Pairwise comparisons show the grid $(1.71 \pm 0.86)$ is significantly less distracting than the foreground guide $(3.08 \pm 1.1 ; \quad p=.001)$. The background guide $(2.33 \pm 0.87)$ is also significantly less distracting than foreground guide $(p=.008)$. In summary, the grid is the most effective guide for direction due to its lower completion time and lowest distraction.

\subsubsection{Distance}

Task 5 tested distance. The results show a significant difference for time $(F(3,69)=7.644$; $p=0.005)$ but none for error rate $\left(x^{2}(3)=2.973\right.$; $p=0.396)$. Pairwise comparisons show that the control condition (21.71s \pm 5.52$)$ results in a significantly lower completion time than grid $(30.63 \mathrm{~s} \pm 10.19 ; \mathrm{p}<0.001)$ and background guide (30.58s $\pm 11.9 ; \quad \mathrm{p}=0.006)$. Similar to the control condition, the foreground guide $(23.17 \mathrm{~s} \pm 6.36)$ is significantly faster than grid $(p=0.008)$ and background guide $(p=0.01)$.

There is no significant difference for helpfulness but one for distraction $\left(X^{2}(2)=9.387 ; p=0.009\right)$. Pairwise comparisons show that the grid $(1.42 \pm 0.58)$ is 


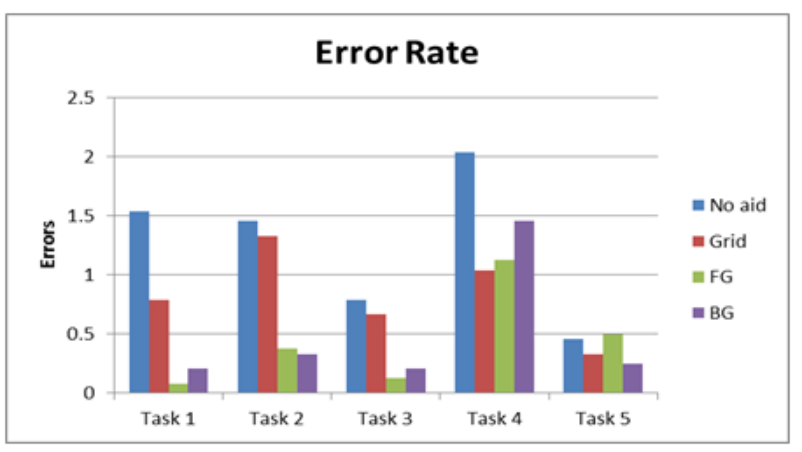

Figure 5: The error rate means for the 5 tasks and the visual support: none, grid, foreground and background.

significantly less distracting than the foreground guide $(2.33 \pm 1.2 ; p=0.003)$. In summary, grid is the most effective guide for distance as it is the least distracting.

The participants final ranking of performance conducted at the study's conclusion shows a significant difference, $X^{2}(3)=49.35 ; \quad p<0.001$. Pairwise comparisons show that no help $(3.96 \pm 0.2)$ is significantly lower ranked than the visual guides. The grid $(2.58 \pm 0.78)$ is also significantly lower ranked than foreground $(1.96 \pm 0.75)$ and background guides $(1.5 \pm 0.66)$, which are equally ranked. For the individual ranking, tasks 1,2 and 3 show the same pattern where the control condition is significantly lower than all the others and the grid is significantly lower ranked as the background and foreground. However, for tasks 4 and 5 there is no significant difference between grid $\left(2 \pm 0.76_{\text {Task } 4}\right.$; $\left.1.83 \pm 0.75_{\text {Task } 5}\right), \quad$ foreground $\quad\left(2.71 \pm 1.21_{\text {Task } 4}\right.$; $\left.2.21 \pm 1.04_{\text {Task5 }}\right)$ and background $\left(1.88 \pm 1.01_{\text {Task } 4}\right.$; $2.25 \pm 0.97_{\text {Task5 }}$ ) with the grid being higher ranked than the foreground guide.

The final rating of the guides over all the tasks shows a significant difference, $X^{2}(3)=46.89$; $p<0.001$. Pairwise comparisons show a similar distribution as the results of the ranking; i.e. the control is the lowest rated $(2.1 \pm 1.83)$, followed by the grid $(5.48 \pm 1.5)$. There is no significant difference between the foreground $(6.83 \pm 1.19)$ and background guides $(7.48 \pm 1.03)$.

\subsection{Participant Comments \& Observations}

At the conclusion of each study we asked the participant to comment on what they liked and disliked about the three visual guides. The grid was described by eight participants as a frame of reference where the vertical and horizontal lines allow for precise judgments of direction and distance. Eleven participants also pointed out the advantages of the grid to gauge the size of objects. However, six participants said they had difficulty identifying a shape with the help of a grid. They commented on how they would try to reverse engineer the visual distortion but would eventually give up. This is consistent with the facilitator's observations and the completion time, which was always higher than the other guides except for task 4.

The foreground guide made comprehending content easy as pointed out by five participants. They stated that identifying shapes was particularly easy due to the preserved shape and the magnified size of the objects. However, seven participants found the foreground distracting. In addition, more than half $(13)$ of the 24 participants asserted their dislike for the foreground guide with the shapes being "always out of focus and literally all over the place" (P4).

The background guide's most commented feature was seeing the shapes in both views as they are drawn (10) - the ink is simultaneously displayed in the magnified region and in the lens background. Eight participants also commented positively on the ease of comprehension of the guide due to the original view being retained under the lens. However, four participants stated their dislike for the clutter and three that the original view was sometimes occluded by the lens content.

\section{$6 \quad$ DISCUSSION}

The background and grid guides are better than the foreground guide and no guide. If all four identifying properties (scale, alignment, direction and distance) have to be equally supported the background guide is most suited as it delivers consistently positive results. However, the grid allows for better comprehension when direction and distance are most important. The foreground, while competitive on the quantitative measures was disliked by users. Any of the visual guides is preferable in quantitative and qualitative measures compared with no guide.

Comparing the background and foreground guides there are few differences in the quantitative measures (completion time, error rate) and helpfulness. However users strongly preferred the background guide, noting two major issues with the foreground guide during the active canvas tasks 4 \& 5: changing focus was reported as being difficult, and the perceived movement of the background was reported to be distracting.

The foreground guide displays the undistorted view away from the lens and thus the center of focus. This required the participants to change their focus between the lens and guide content and thus results in a higher mental workload [2]. Having to change focus like this is similar to using a radar window where one also has to switch between views. However, the foreground guide has the advantage of both views being integrated thus making better use of the total space available. The distraction factor was the other complaint of the foreground guide. This is caused by the movement 
of the foreground over the canvas as the lens moves. Since this additional information is outside the lens it seems as if everything is moving. In contrast the background guide allowed the user to fully concentrate on the lens by displaying the additional undistorted view of the distorted shapes in the lens's background at its original size and location. Additionally with the background guide participants took advantage of shapes appearing in the background at their original size and position as they were drawing.

Quantitative results suggest judgments of alignment and scale are best supported by the foreground and background guides. This includes the identification of distorted shapes. However the foreground guide has the distraction problems discussed above, making the background guide the better guide for alignment and scale.

The grid was best for judging direction and distance. Participants used the grid lines as a frame of reference allowing them to accurately estimate direction and distance by following the lines. Supporting our results, other researchers have successfully used grids in distorted visualizations for tasks where judging direction and distance is most important such as geographic maps (Zanella, Carpendale et al. 2002). In non-distortion based environments the conditions are different, in that no mental reverse engineering of the distorted content, including the distorted gridlines, is required. Interestingly using the grid as a frame of reference is unsuitable for judging alignment and scale for visual distortions. The participants disappointment was reflected in the grid's overall rating and ranking were it is ranked third.

The control condition was consistently perceived as the least preferred condition across all measurements. The lack of distraction and shorter completion time are the only advantages of having no visual support. As there is no additional information displayed, the participants had no extra information to incorporate in their decision. However the results also showed that except for judging direction having no help always resulted in significantly higher error rates. It was always the least helpful and least preferred condition and was consistently rated and ranked the lowest.

The ordering of the orientating properties is different from Cleveland and McGill (1984), Wigdor, Shen at al. (2007) and Bezerianos and Isenberg (2012) in that scale (or area) judgments produced the least errors followed by distance, angle and direction. A reason could be that participants in our experiment were required to order them relative to their size and not apply absolute sizes as done by the other authors. If only the error percentage is considered when people had to match the magnified but undistorted object with its distorted version of equal size, then the order of properties is identical to Cleveland and McGill's results with scale being the least accurate.

The interactive sketching context was used to gauge the effects of distortions on information and evaluate our two novel visual guides because it is an easy and familiar metaphor to create and edit content. Editing is important to target the individual orientation properties especially distance and direction. To apply the proposed guides to other domains (e.g. map navigation (Pattath, Ebert et al. 2009)) adjustments may be required. Rather than using one translucent layer Lieberman (1994) used several translucent layers to accommodate information layers for browsing an image or a map. Similarly, Pietriga and Appert (2008) used one translucent layer but with varying levels of translucency to transition between focus and context areas in distortion lenses. Exploring other contexts is a subject for future work.

\section{CONCLUSION \& FUTURE WORK}

Displaying visually distorted information has a number of disadvantages such as higher mental workload and comprehension difficulties, but has the advantage of non-occluding magnification. We developed and implemented two novel visual guides and evaluated them. We specifically looked at the four disrupted orientation properties important for identifying distorted information: scale, alignment, distance and direction.

Our introductory research questions were (a) the effect of distortions on comprehension and (b) the degree to which visual guides can aid comprehension. Based on the results visual changes in the distortion region made information comprehension considerably more difficult as the control condition had a higher error rate and lower user preference compared to the tested guides. The grid and background guide both aided comprehension without distracting the user.

We propose the following three guidelines for the best interaction when designing distortion based magnification lenses: First, any visual support is better than none. Second, overall the background lens is most suitable. However, third, the grid is more appropriate when precise judgments involving only direction and distance are required.

While there is no guide outperforming the other guides for all identifying properties, a combination of the background and grid may be more suitable than either individually. There is a danger of such a combination being too distracting; we plan to study this combination in future work. The final ratings show that while none of the guides are yet perfect, our novel background guide is overall best. 


\section{REFERENCES}

Agrawala, M. and M. Shilman. DIZI: A Digital Ink Zooming Interface for Document Annotation INTERACT 2005, Springer (12-16 Sep), 69-79.

Bederson, B. B. Fisheye Menus. UIST 2000, ACM (5-8 Nov), 217-225.

Bertin, J. (1977).La graphique et le traitement graphique de l'information. Flammarion Paris.

Bezerianos, A. and P. Isenberg. (2012) Perception of Visual Variables on Tiled Wall-Sized Displays for Information Visualization Applications. IEEE Transactions on Visualization and Computer Graphics, 18.

Brosz, J., S. Carpendale and M. A. Nacenta. (2011) The Undistort Lens. Computer Graphics Forum, 30, 881-890.

Carpendale, M. S. T., D. J. Cowperthwaite and F. D. Fracchia. Making Distortions Comprehensible. Visual Languages, 1997. Proceedings. IEEE Computer Society (23 -27 Sep), 36-45.

Cleveland, W. S. and R. McGill. (1984) Graphical Perception: Theory, Experimentation, and Application to the Development of Graphical Methods. Journal of the American Statistical Association, 79, 531-554.

Cleveland, W. S. and R. McGill. (1985) Graphical Perception and Graphical Methods for Analyzing Scientific Data. Science, 229, 828-833.

Cockburn, A., A. Karlson and B. B. Bederson. (2009) A Review of Overview+Detail, Zooming, and Focus+Context Interfaces. ACM Comput. Surv., 41, 1-31.

Cockburn, A., J. Savage and A. Wallace. Tuning and Testing Scrolling Interfaces that Automatically Zoom. Proceedings of the SIGCHI conference on Human factors in computing systems 2005, ACM (2-7 Apr), 71-80.

Furnas, G. Generalized Fisheye Views. Proceedings of the SIGCHI conference on Human factors in computing systems 1986, ACM (13-17 Apr), 16-23.

Grudin, J. Partitioning Digital Worlds: Focal and Peripheral Awareness in Multiple Monitor Use. Proceedings of the SIGCHI conference on Human factors in computing systems 2001, ACM (1-5 Apr), 458-465.

Gutwin, C. Improving Focus Targeting in Interactive Fisheye Views. CHI 2002, ACM (20-25 Apr), 267274.

Gutwin, C. and A. Skopik. Fisheyes are Good for Large Steering Tasks. Proceedings of the SIGCHI conference on Human factors in computing systems 2003, ACM (5-10 Apr), 201-208.

Hornbaek, K. and E. Frokjaer. (2003) Reading Patterns and Usability in Visualizations of Electronic Documents. ACM Trans. Comput.-Hum. Interact., 10, 119-149.

Jakobsen, M. R. and K. Hornbaek (2011). Sizing Up Visualizations: Effects of Display Size in Focus+Context, Overview+Detail, and Zooming
Interfaces. Proceedings of the 2011 annual conference on Human factors in computing systems. Vancouver, BC, Canada, ACM: 14511460.

Jul, S. and G. W. Furnas (1998). Critical Zones in Desert Fog: Aids to Multiscale Navigation. Proceedings of the 11th annual ACM symposium on User interface software and technology. San Francisco, California, United States, ACM: 97-106. Kosara, R., S. Miksch and H. Hauser. Semantic Depth of Field. INFOVIS 2001, IEEE Computer Society (21-26 Oct), 97.

Lank, E. and S. Phan. Focus+Context Sketching on a Pocket PC. $\mathrm{CHI}$ '04 extended abstracts on Human factors in computing systems 2004, ACM (24-29 Apr), 1275-1278.

Lieberman, H. (1994). Powers of Ten Thousand: Navigating in Large Information Spaces. Proceedings of the 7th annual ACM symposium on User interface software and technology. Marina del Rey, California, United States, ACM: 15-16.

Pattath, A., D. S. Ebert, W. Pike and R. A. May. Contextual Interaction for Geospatial Visual Analytics on Mobile Devices. 2009, 14.

Pietriga, E. and C. Appert. Sigma Lenses: FocusContext Transitions Combining Space, Time and Translucence. Proceeding of the twenty-sixth annual SIGCHI conference on Human factors in computing systems 2008, ACM (5-10 Apr), 13431352.

Ramos, G., A. Cockburn, R. Balakrishnan and M. Beaudouin-Lafon. Pointing lenses: facilitating stylus input through visual-and motor-space magnification. 2007, ACM 757-766.

Sarkar, M. and M. Brown. Graphical Fisheye Views of Graphs. 1992, ACM 83-91.

Schmieder, P., B. Plimmer and J. Hosking (2012). Non-Occluding Intelligent Magnifiers for Sketching on Small Displays. HCl. Birmingham, England.

Wagner, M. (2006). The Geometries of Visual Space. Lawrence Erlbaum Associates, Mahwah, NJ, USA.

Wickens, C. D. and J. G. Hollands. (2000). Decision Making. Engineering Psychology and Human Performance. Prentice Hall, Upper Saddle River, NJ, USA.

Wigdor, D., C. Shen, C. Forlines and R. Balakrishnan (2007). Perception of Elementary Graphical Elements in Tabletop and Multi-Surface Environments. Proceedings of the SIGCHI Conference on Human Factors in Computing Systems. San Jose, California, USA, ACM: 473482.

Zanella, A., M. S. T. Carpendale and M. Rounding (2002). On the Effects of Viewing Cues in Comprehending Distortions. Proceedings of the second Nordic conference on Human-computer interaction. Aarhus, Denmark, ACM: 119-128. 